\title{
CARRIER TUNNELING IN HIGH MAGNETIC FIELDS
}

\author{
P.C.M. Christianen, I.E.M. Bruggink, J.C. MaAN
}

High Field Magnet Laboratory and Research Institute for Materials University of Nijmegen, Toernooiveld, 6525 ED Nijmegen, The Netherlands

\section{AND W. VAN DER VLEUTEN}

COBRA Interuniversity Research Institute, Eindhoven University of Technology P.O. Box 513, 5600 MB Eindhoven, The Netherlands

A magnetic field induced coupling is observed between the Landau levels with different quantum number of two GaAs quantum wells separated by a thin $(\mathrm{Ga}, \mathrm{Al})$ As tunnel barrier using magneto-photoluminescence-excitation spectroscopy. This coupling cannot be due to a resonance between single particle energy levels, and therefore an explanation is proposed in terms of the Coulomb interaction of magneto-excitons across the thin barrier.

PACS numbers: 71.35.tz, 73.20.Dx, 78.20.Ls

\section{Introduction}

In an asymmetric double quantum well (ADQW) structure, consisting of two quantum wells (QW's) of different well width separated by a thin barrier, the tunneling of carriers from one well to the other can be detected directly by means of optical spectroscopy. The basic question whether electrons and holes tunnel independently or as bound electron-hole pairs is still a matter of controversy. For instance in GaAs based ADQW's it has been found that, although the carriers tunnel independently, the Coulomb interaction between electrons and holes strongly affects the tunneling behaviour [1]. Furthermore, Lawrence et al. [2] have recently claimed that in CdTe based ADQW's the dominant transfer mechanism is associated with the tunneling of an exciton as a whole, which is favoured by the enhanced exciton binding energy in that material. Here we study the coupling between two GaAs QW's in high magnetic fields, directed perpendicular to the plane of the QW's, and therefore the tunneling occurs between quantized Landau levels. In a single particle picture, because of momentum conservation, only tunneling between the Landau levels of equal quantum number is allowed. Experimental evidence presented here shows a coupling between the Landau levels with different quantum number, indicating the importance of the Coulomb interaction of magneto-excitons in the neighbouring QW's. 


\section{Experimental results}

The MBE grown ADQW structures contain, apart from the usual buffer and cap layers, ten double quantum well units decoupled by $200 \AA(\mathrm{Ga}, \mathrm{Al}) \mathrm{As}$ layers. Each unit consists of a wide well (WW) of $100 \AA$ and a narrow well (NW) of $50 \AA$ thickness separated by a thin $(\mathrm{Ga}, \mathrm{Al})$ As barrier of variable thickness $(40$ or $80 \AA$ ). All experiments were repeated on a control sample, consisting of one unit of two quantum wells of $100 \AA$ and $70 \AA$ width decoupled by a $500 \AA(\mathrm{Ga}, \mathrm{Al}) \mathrm{As}$ barrier. The $\mathrm{Al}$ content of the $(\mathrm{Ga}, \mathrm{Al})$ As layers in all samples equals $35 \%$. Magneto-photoluminescence excitation (M-PLE) spectra were recorded at $4.2 \mathrm{~K}$ using a tunable Tisapphire laser, a single grating monochromator and a cooled GaAs detector. Magnetic fields up to $17 \mathrm{~T}$ were used, with the field oriented along the growth axis. Typical spectra for the sample with $40 \AA$ barrier are depicted in Fig. 1. At finite fields clear Landau level transitions appear in the spectra, shifting up in energy with field $B$ according to the Landau level quantization: $E_{N}=\left(N+\frac{1}{2}\right) \hbar e B / \mu^{*}\left(N\right.$ is the Landau level index, $\mu^{*}$ is the reduced effective mass). Figure 2 shows the resulting fan chart for the $40 \AA$ barrier

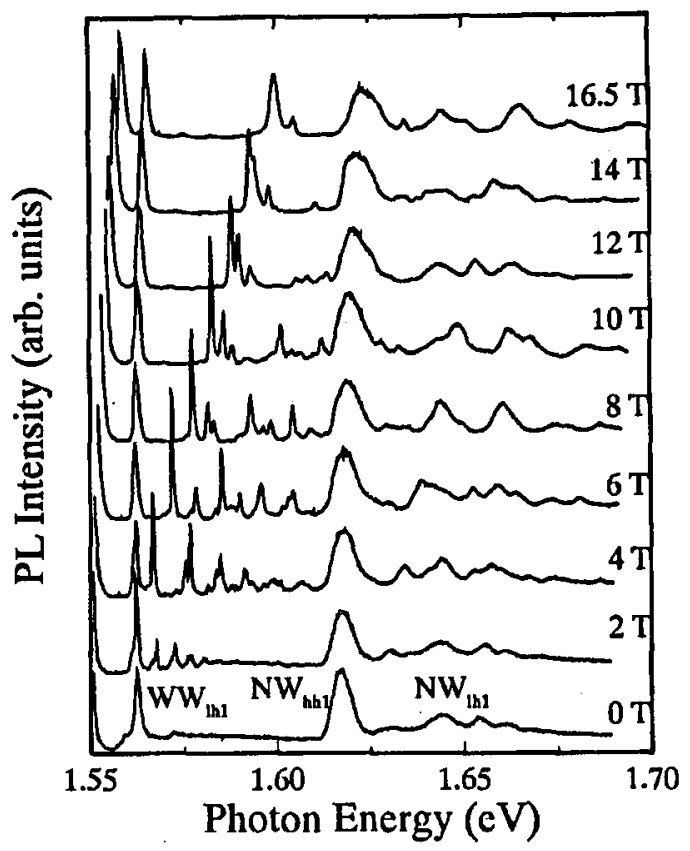

Fig. 1. Typical M-PLE spectra for the ADQW structure with $40 \AA$ barrier. All curves were measured with the detection wavelength at the peak of the WW heavy hole exciton emission. At zero magnetic field transitions are observed due to the wide well light hole exciton $\left(\mathrm{WW}_{\mathrm{lh} 1}, 1.563 \mathrm{eV}\right)$, the narrow well heavy hole exciton $\left(\mathrm{NW}_{\mathrm{hh} 1}, 1.617 \mathrm{eV}\right)$, and the narrow well light hole exciton $\left(\mathrm{NW}_{\mathrm{th} 1}, 1.645 \mathrm{eV}\right)$. 


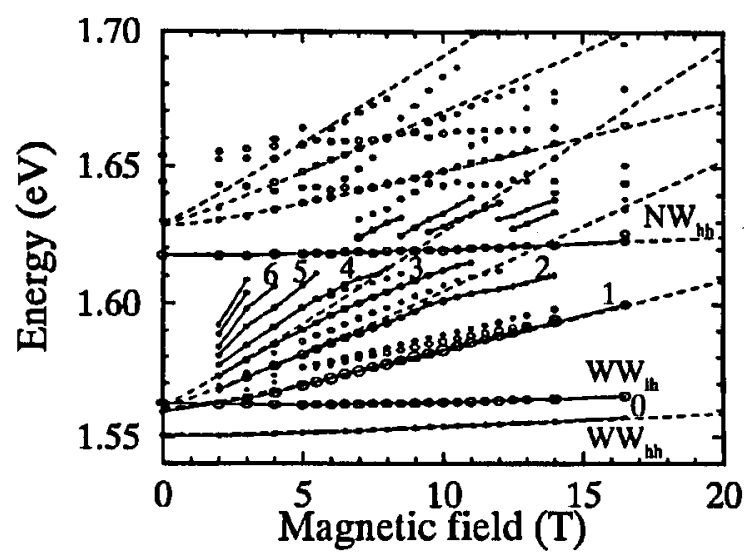

Fig. 2. Landau level fan diagram of the $40 \AA$ barrier ADQW. The symbols represent the energy positions of the optical transitions, and their size corresponds to the transition strength. The solid curves interconnect the data points for the $\mathrm{WW}_{\mathrm{hh}}$ Landau level transitions, where the numbers reflect the Landau level indices. The dashed curves are calculated by solving the Schrödinger equation for a two-dimensional exciton in a magnetic field.

sample. Focusing on the magnetic field dependence of the $W_{\mathrm{hh}}$ Landau levels, in the vicinity of the $\mathrm{NW}_{\mathrm{hh}}(N=0)$ peak, we interconnected the relevant data points by solid lines in Fig. 2. The dashed lines were calculated by solving Schrödinger's equation of a two-dimensional exciton in a magnetic field [3, 4]. These dashed curves describe the data points fairly well, except for the $W_{\text {hh }}$ higher index curves $(N \geq 2)$, where the experimental points deviate from the model when approaching the lowest $\mathrm{NW}$ transition $(N=0)$. Similar results were obtained for the $80 \AA$ barrier sample, whereas the results on the control sample were entirely different. In this case the spectra of the $100 \AA \mathrm{QW}$ showed no influence from the neighbouring $70 \AA \mathrm{QW}$.

\section{Discussion}

In a magnetic field the in-plane Landau level quantization is strictly decoupled from the spatial confinement in the growth direction. Thus no coupling occurs even in the case of an energetic resonance between WW and NW Landau levels of different quantum number (because of momentum conservation) [5]. Therefore we cannot explain the experimentally observed interwell coupling in a single particle picture, and an alternative coupling mechanism is necessary to produce a mixing of the Landau quantization and the spatial QW confinement. Because the exciton Bohr radius is larger than the well separation, the Coulomb interaction is a good candidate. For barriers of only 40 or $80 \AA$ the Coulomb field of an exciton in one well is still very effective in the other well. Recent experiments on carrier tunneling in GaAs based ADQW's prove that interwell excitonic effects are very important [1]. In a field it is likely that excitonic effects will grow in importance 
because with increasing magnetic field the exciton binding energy is enhanced considerably, due to the Lorentz force induced reduction of the in-plane exciton radius [3]. Actually, Lawrence et al. [2] observed tunneling of magneto-excitons in CdTe based ADQW's, due to the increased exciton binding energy in a magnetic field, in addition to the enhanced binding energy in these kind of materials. In view of these considerations we postulate the observation of coupling of the Landau levels with different quantum number mediated by the Coulomb interaction of magneto-excitons across the thin barrier.

\section{References}

[1] R. Ferreira, P. Rolland, Ph. Roussignol, C. Delalande, A. Vinattieri, L. Carraresi, M. Collocci, N. Roy, B. Sermage, J.F. Palmier, B. Etienne, Phys. Rev. B 45, 11782 (1992).

[2] I. Lawrence, S. Haacke, H. Mariette, W.W. Rühle, H. Ulmer-Tuffigo, J. Cibert, G. Feuillet, Phys. Rev. Lett. 73, 2131 (1994).

[3] J.C. Maan, in: The Physics of Low-Dimensional Semiconductor Structures, Eds. P. Butcher, N.II. March, M.P. Tosi, Plenum Press, New York 1993, p. 333.

[4] A.H. MacDonald, D.S. Ritchie, Phys. Rev. B 33, 8336 (1986).

[5] E.E. Mendez, L. Esaki, W.I. Wang, Phys. Rev. B 33, 2893 (1986). 